\title{
COVID-19 pandemic and transfusion medicine: the worldwide challenge and its implications
}

\author{
Layal AI Mahmasani ${ }^{1} \cdot$ Mohammad Hassan Hodroj $^{1} \cdot$ Antoine Finianos $^{2} \cdot$ Ali Taher $^{2}$
}

Received: 19 December 2020 / Accepted: 26 January 2021 / Published online: 1 February 2021

(C) The Author(s), under exclusive licence to Springer-Verlag GmbH, DE part of Springer Nature 2021

\begin{abstract}
COVID-19 pandemic has imposed worldwide challenge and has significantly affected transfusion medicine. Shortage in blood products along with concerns regarding the safety of blood products have emerged. Measures to overcome these challenges have been implemented in order to decrease the demand on blood products and to encourage blood donations while taking full precautions to minimize risk of COVID-19 transmission mainly at blood banks and medical centers. Several countries have been successful in facing these new challenges. In addition, the role of plasma therapy in the treatment of COVID-19 patients, especially in severe cases, has been proposed and current studies are being conducted to determine its efficacy. Other therapeutic options are currently being explored. So far, the use of convalescent plasma is considered a promising rescue treatment to be looked at.
\end{abstract}

Keywords COVID-19 $\cdot$ Transfusion $\cdot$ Blood safety $\cdot$ Shortage $\cdot$ Plasma therapy

\section{Introduction}

\section{Overview of coronaviruses}

Coronaviruses (CoVs) are a subfamily of large and enveloped viruses that are divided based on their genome characteristics into four genera: alpha, beta, gamma, and delta-CoV, of which alpha and beta-CoVs are known to infect humans [1]. All human CoVs appear to have respiratory transmission carrying a pandemic potential and are believed to be zoonotic in origin with bats being most likely the natural hosts for all presently known CoVs [2]. Six coronaviruses were known to cause disease in humans, two among them, severe acute respiratory syndrome coronavirus (SARS-CoV) and Middle East respiratory syndrome coronavirus

Layal Al Mahmasani and Mohammad Hassan Hodroj contributed equally to this work.

Ali Taher

ataher@aub.edu.lb

1 Department of Internal Medicine, American University of Beirut Medical Center, Beirut, Lebanon

2 Department of Internal Medicine, Division of Haematology-Oncology, American University of Beirut Medical Centre, Cairo Street, PO Box 11-0236, Riad El Solh, Beirut 1107 2020, Lebanon
(MERS-CoV), have caused more widespread concern resulting in epidemics with significant morbidity and mortality [3]. SARS$\mathrm{CoV}$ emerged in 2002 in the Guangdong province of China with a mortality rate of $10 \%$ [4], whereas MERS-CoV hit in 2012 in Saudi Arabia exhibiting a high case fatality ratio of $35 \%$ making it one of the deadliest human pathogens [5].

\section{Novel coronavirus pandemic and epidemiology}

Late in December 2019, a novel coronavirus was identified to be responsible for unidentified pneumonia outbreaks and sporadic human infections that emerged in Wuhan, Hubei Province, China [6]. It was labeled as the seventh type of coronavirus to infect humans and the third highly pathogenic human $\mathrm{CoV}$ in this century with a remarkable rapid pace of transmission [7]. In addition, phylogenetic analysis showed that the genomic sequence of the current virus is closer to that of SARS$\mathrm{CoV}$ than that of MERS-CoV for which it was given the nomenclature SARS-CoV-2 the causative pathogen of coronavirus disease 2019 (COVID-19) as the standard format announced by the World Health Organization (WHO) $[8,9]$.

Clinically, COVID-19 has an incubation period of 1 to 14 days mostly ranging between 3 and 7 days with respiratory droplets and close contact being the main routes of 
transmission. The SARS-CoV-2 infection can induce five different outcomes in a spectrum that ranges from being asymptomatic $(1.2 \%)$, mild to moderate cases $(80.9 \%)$, severe cases (13.8\%), and reaching critical cases $(4.7 \%)$ and death in $2.3 \%$ of all reported cases [10]. The most prominent symptoms in mild to moderate patients are fever, fatigue, and dry cough, followed by other symptoms such as sore throat, myalgia, arthralgia, and headache [11]. As disease progresses, multiple complications tend to develop, especially in critically ill patients admitted to the ICU including, shock, sepsis, acute kidney injury, acute cardiac injury, and multi-organ dysfunction [12]. COVID-19 rapidly progressed and spread across various continents over few months and was formally declared as pandemic by WHO on March 11, 2020 [13].

\section{Transfusion medicine and possible pandemic drawbacks}

Transfusion medicine is a vital multidisciplinary branch of medicine that is specialized in the proper use and management of blood and blood products for treatment or prevention of diseases [14]. Since its foundation by Karl Landsteiner who discovered the ABO system in 1902, the field experienced massive progressive development in terms of safety measures, storage, and administration techniques. However, it has also faced several challenges in overcoming infection transmission, especially with the emergence of different blood-borne diseases [15]. COVID-19 constitutes a new challenge for the transfusion sector with preliminary data indicating the presence of viremia in $15 \%$ of the COVID-19 patients which means that the risk of transmitting the virus through transfusions cannot be excluded despite the fact that the detected viral RNA concentrations in blood are low [16]. Viral RNA requires at least 2 to 3 days after the onset of symptoms to be detectable in plasma or serum. Moreover, most patients, especially younger adults who can donate blood, are either asymptomatic or have mild symptoms increasing the possibility of carrier patients donating blood [17]. Furthermore, samples collected from patients who recovered from COVID-19 showed positive reverse transcriptase-polymerase chain reaction (RT-PCR) tests which have not been seen before in the human infectious diseases' history [18]. COVID-19 pandemic had affected the field of transfusion in many aspects threatening its efficiency and safety through possible drawbacks including blood shortage due to decreased donations, increased demand in intensive care units, and risk of the virus transmission [19].

The current review investigates the effects of COVID19 on transfusion medicine and the safety measures taken to face the current pandemic. It also discusses the role of plasma therapy as an option in the treatment of COVID-19 patients.

\section{The challenge and blood safety}

\section{Inactivation of COVID-19 in blood products}

Coronaviruses are enveloped viruses that are vulnerable to acid-pH, basic-pH, and heat which works mainly on denaturing the secondary structure viral proteins. The virus seems to be stable at a temperature of $4{ }^{\circ} \mathrm{C}$ and below without any significant reduction in the viral infectious titer after 25 cycles of freezing and thawing [20]. Following the outbreaks of SARS and MERS, some studies were conducted to investigate and develop pathogen inactivation/reduction technologies (PRTs) to decrease or completely eradicate the potential risk of coronavirus transmission via blood products. Several techniques were studied including heat, solvent and detergent treatment, methylene blue, and illumination by ultraviolet (UV) light all of which have shown an ability of virus inactivation of variable extents [17]. Previous studies revealed that a temperature of $60^{\circ} \mathrm{C}$ for $15-30 \mathrm{~min}$ is enough for reduction of SARS-CoV from plasma without cells, whereas the inactivation in plasma products can be achieved by heating with $60^{\circ} \mathrm{C}$ for $10 \mathrm{~h}[17,21]$. However, heating can only be used in manufactured plasma-derived products since it can denature proteins in blood products. This fact stimulated the search for other practical techniques where solvent and detergent method, using detergent-like solutions such as Triton X-100 and Tween, was found to be effective [17]. Additionally, different wavelengths of UV light have influenced the activities of both SARS-CoV and MERS-CoV in the blood. UV-A and UV-B lights were used in the presence of co-factors as riboflavin to inactivate the pathogens' nucleic acids, while UV-C light was used solely [17]. On the contrary, high cost remains the major administrative obstacle to the practical implementation of PRTs [22].

\section{Biosafety and screening strategies}

Besides the classical public health measures to control the pandemic including isolation, quarantine, social distancing, and community containment, it was essential to have specific measures for the field of transfusion [23]. COVID-19 pandemic directly affected different phases of the transfusion process mainly the pre-transfusion examination in laboratories besides the clinical transfusion phase. In order to reduce the risks of COVID-19 transmission through transfusions, urgent adjustments to the existing biosafety protocols were made by various international and local health organizations [24]. Donor screening and testing strategies are usually based on theoretical or confirmed risks of transmission through transfusions. Since SARS-CoV-2 is still a new virus, its transmission through a viraemic donor is uncertain, which leads the current knowledge regarding this issue to be based on the 
experience from other coronaviruses where the risk of transmission through blood is considered low $[25,26]$.

In addition to the local Clinical and Diagnostic Centers in China and many other countries, the World Health Organization was a pioneer in formulating a batch of updated laboratory biosafety guidelines specific for COVID-19 [19]. These guidelines include a group of protection measures that are aimed at minimizing the risk of virus spread via transfusions or laboratory procedures and protecting both the laboratory personnel and the patients in a studied and organized pattern which maintains the continuity of safe blood donations. The recommendations focus on performing a local risk assessment by each process step starting from sample collection and sample reception, until reaching clinical testing and PCR [27]. The issue of wearing proper personal protective equipment was also explained thoroughly with insisting on the importance of hand washing, use of disinfectants as $75 \%$ ethanol spraying, and biological waste management. Moreover, the new guidelines highlight the importance of conducting manual procedures in biological safety cabinets to reduce the risks of contamination. These guidelines and protocols are progressively updated and improved based on the latest research studies and information about the virus and the pandemic to be able to cope properly with the current situation $[27,28]$.

\section{Impact on practice}

In general, the challenges faced by blood banks during an infectious pandemic are securing and protecting the blood supply [29]. Historically, during a pandemic, the demand for blood and blood products may decrease due to postponement of elective surgeries or due to measures such as physical distancing and complete lockdown of cities or countries in an attempt to curb the spread of infection may result in a large decline in blood supply and an overall shortage of blood products [30]. In addition, due to the nature of COVID-19 virus, which has long incubation period and the possibility of having asymptomatic carriers of the virus, a huge challenge in the recruitment of blood donors, blood collection, and blood safety is inevitable, and a profound impact on the number of blood donations, blood supplies, and blood safety has been experienced since the start of the outbreak [19].

The main reason behind the reduction in blood products is related to the COVID-19 precautions issued by governments and social media to avoid crowds and unnecessary commuting [31]. The effect of these measures taken was noted in several countries and blood centers. For example, in Italy, it has been reported that in the weeks 2-8 of March, 44,297 whole blood units were collected and 46,183 red cell units were transfused, with a negative balance of 1886 units as reported by the Italian National Blood Center [16]. In the USA, nearly 4000 American Red Cross blood drives have been canceled across the country and hospital-based collections have been canceled due to institutional concerns regarding donors spreading COVID-19 to hospitalized patients or vice versa [32]. Due to the anticipated shortage of blood supply, measures were taken to avoid overwhelming the system and this, in turn, resulted in decrease in the total number of blood products transfused. For instance, at the HealthCity Novena campus, a mean of 1270 packed red blood cell (pRBC) units/month in 2019 were transfused, as compared to 1063 PRBC units/ month (16\% decrease) in February and March 2020 in addition to reduction in fresh frozen plasma (FFP) from 245 to 193 units/month (21.2\% decrease) and platelet products from 197 to 166 units/month (15.7\% decrease) [30]. Hence, one of the major implications of COVID-19 pandemic is the significant reduction in blood donations compared to previous years resulting in clinical shortage of blood supply and a worrying decrease in the number of blood donations [16].

\section{Blood transfusion demand in COVID-19 patients}

Whether patients with COVID-19 infection have a higher need for blood transfusion or not is a reasonable question given the shortage in blood supply. From the data available so far as the pandemic continues, it has been shown that patients infected with COVID-19 who are critically ill are those who might require transfusion of blood product. A study by Bingwen et al. showed that 9 out of 572 patients with COVID19 required transfusion: $0.63 \%$ of non-ICU (intensive care unit) COVID-19 patients compared to $36.8 \%$ of ICU COVID-19 patients requiring pRBC transfusion, with lesser requirements for FFP and platelet transfusion [30]. The reasons behind the need for $\mathrm{pRBC}$ transfusion were mainly related to either severe gastrointestinal bleeding or symptomatic anemia in premenopausal women with iron deficiency anemia that have concurrent COVID-19 infection rather than hemolysis [30]. In the latter group of premenopausal women, it is worth noting that alternative approach with iron replacement therapy aiming at improving erythropoiesis was considered a more appropriate treatment approach. To date, most patients with COVID-19 infection do not require blood transfusion and only a subset of critically ill patients in the ICU require blood transfusion especially in the setting of gastrointestinal bleeding [30].

\section{Measures taken by blood centers and hospitals}

In face of COVID-19 pandemic, several measures are taken to overcome the emerging shortage in blood products. These measures vary from one country to another and from one blood center to another. Examples of measures taken in three different countries (China, Iran, and Italy) are presented in Table 1. In general, the measures taken can be divided into two main arms: (1) limiting the spread of COVID-19 and (2) 
Table 1 Examples of measures taken in 3 different countries (China, Iran, and Italy)

\begin{tabular}{|c|c|c|}
\hline China [32] & Iran [33] & Italy [29] \\
\hline $\begin{array}{l}\text { Pleading the public for } \\
\text { blood donations, } \\
\text { instead of having } \\
\text { volunteer donors at } \\
\text { blood drives }\end{array}$ & $\begin{array}{l}\text { Creating online } \\
\text { system for } \\
\text { coordination among } \\
\text { blood centers }\end{array}$ & $\begin{array}{l}\text { Reorganizing hospital } \\
\text { activities }\end{array}$ \\
\hline $\begin{array}{l}\text { Recruiting donors } \\
\text { through traditional } \\
\text { and social media }\end{array}$ & $\begin{array}{l}\text { Ensuring enough } \\
\text { personal protective } \\
\text { equipment for } \\
\text { employees and } \\
\text { donors }\end{array}$ & $\begin{array}{l}\text { Running national } \\
\text { media campaigns to } \\
\text { increase awareness } \\
\text { on blood donation }\end{array}$ \\
\hline $\begin{array}{l}\text { Providing donors with } \\
\text { information about } \\
\text { COVID-19 before } \\
\text { hand and taking ap- } \\
\text { pointments to donate } \\
\text { blood }\end{array}$ & $\begin{array}{l}\text { Changing the style of } \\
\text { waiting line chairs } \\
\text { at donation sites }\end{array}$ & $\begin{array}{l}\text { Deferring donors who } \\
\text { had fever*, } \\
\text { symptoms of } \\
\text { respiratory tract } \\
\text { infection**, or who } \\
\text { have had contact } \\
\text { with a suspected or } \\
\text { confirmed case of } \\
\text { COVID-19 within } \\
\text { the past } 2 \text { weeks of } \\
\text { donation }\end{array}$ \\
\hline $\begin{array}{l}\text { Deferring blood } \\
\text { donations from } \\
\text { donors traveling } \\
\text { from, or residing in, } \\
\text { the regions hardest } \\
\text { hit by COVID-19 }\end{array}$ & $\begin{array}{l}\text { Increasing disinfection } \\
\text { of all the contacting } \\
\text { surfaces }\end{array}$ & \\
\hline $\begin{array}{l}\text { Performing } \\
\text { pre-donation screen- } \\
\text { ing (temperature } \\
\text { check, physical ex- } \\
\text { am) }\end{array}$ & $\begin{array}{l}\text { Decreasing waiting } \\
\text { time through an } \\
\text { online ticketing } \\
\text { system }\end{array}$ & \\
\hline $\begin{array}{l}\text { Thoroughly cleaning } \\
\text { and disinfecting all } \\
\text { sites of blood } \\
\text { donation }\end{array}$ & $\begin{array}{l}\text { Increasing working } \\
\text { hours of donation } \\
\text { centers and } \\
\text { removing weekend } \\
\text { holidays }\end{array}$ & \\
\hline $\begin{array}{l}\text { Providing full } \\
\text { equipment and } \\
\text { protection gear to } \\
\text { laboratory staff }\end{array}$ & & \\
\hline
\end{tabular}

*Fever defined as temperature $>37.5^{\circ} \mathrm{C}$

**Cough, dyspnea, sore throat, rhinorrhea

overcoming the shortage in blood supply. Regarding the first arm, common precautions focusing on ensuring appropriate social distancing, screening donors for COVID-19 symptoms, recent travel or exposure to confirmed cases, and hospital measures focusing on postponing elective procedures have been implemented. As for plans to overcome the shortage in blood supply, measures focusing on encouraging blood donations through mobile blood drives, social media or traditional media [19], organizing national media campaigns on the importance and safety of blood donation [16], or creating an online system that shows all blood unit inventory in a realtime setting whereby any center with a shortage of supply is able to ask its richest neighbor for units [31] have been applied.

Another approach to overcome the shortage was mainly related to the practice of medicine and more specifically transfusion medicine where calling for restrictive blood transfusion strategies became essential. This is mainly applicable in cancer patients and in ECMO patients where transfusion protocols with lower triggers coupled with blood preservation strategies such as auto-transfusion of circuit blood during decannulation would result in reduction of the number of blood transfusions needed [34]. Despite the fact that optimal red cell transfusion thresholds in patients with hematological malignancies have not yet been established [35] and that current practice varies widely [36], restrictive red cell transfusion strategies is still recommended in these patients. In other patients, the use of iron, folic acid, vitamin B12, or erythropoietin may present alternatives to red cell transfusion or could be used to limit transfusion requirement even in the preoperative patients and in the critically ill, where the use of erythropoiesis-stimulating agents at either 100,000 units weekly in the intensive care unit (ICU) or 600 units $/ \mathrm{kg}$ in the preoperative period results in higher hemoglobin concentrations and reduced transfusion utilization [37, 38]. As for platelets which are known to have the shorter shelf-life, international guidelines recommend not to give prophylactic platelet transfusion for asymptomatic patients with chronic bone marrow failure (including patients taking low-dose oral chemotherapy or azacitidine) and to consider not giving prophylactic platelet transfusions to well patients without evidence of bleeding after an autologous stem cell transplant [39]. Other approaches are also recommended depending on the medical condition. For example, in sickle cell disease patients, immediate initiation of low-dose hydroxyurea therapy (fixed dose $10 \mathrm{mg} / \mathrm{kg} /$ day) for all children receiving blood transfusion therapy for primary and secondary stroke prevention has been recommended during COVID-19 pandemic where blood supply interruptions are likely to occur [40].

Transfusion-sparing strategies, such as implementation of patient blood management (PBM) which is an evidence-based bundle of care to optimize medical and surgical patient outcomes by clinically managing and preserving a patient's own blood or the application of medical concepts designed to maintain hemoglobin concentration, optimize hemostasis, and minimize blood loss, in an effort to improve patient outcomes have been suggested and according to some references should be mandated $[32,41]$. There are numerous modalities available for perioperative blood conservation including the use of topical or stimulating agents, avoiding hemodilution and early treatment of coagulopathy [42]. Furthermore, conservative transfusion strategies such as target hemoglobin level of $7-8 \mathrm{~g} / \mathrm{dL}$, increased to $10 \mathrm{~g} / \mathrm{dL}$ if hypoxemia persisted, have been proposed to be applied for COVID-19 patients [43]. Following restrictive blood transfusion strategies coupled with timely and accurate communication among blood centers/hospitals, ensuring that blood 
collection meets the clinical needs and abiding by international guidelines to minimize transmission are key elements in overcoming the challenge.

The results of applying these measures have been proven effective in some countries. For example, in Italy, an increase in the number of collected whole blood units $(53,538$ whole blood units) and a decrease in red blood cell transfusion to 39,745 units (positive balance of 13,793 units) 1 week after running a national media campaign on the importance and safety of blood donation and reorganization of hospital activity were noted [16]. The change was also reported in Iran where it has been reported that after implementing the crisis system for COVID-19, the mean number of donations increased between March 7 and March 17 to 4513.72-1596 donations as compared to weeks prior to implementation of new measures between February 25 and March 6 when the mean number of donations per day was 2828.45-1587 [31]. The same trend was noted in the USA, where during the first week of the outbreak, a significant drop in blood donations occurred, but subsequently, blood units were provided from blood centers of non-affected areas of the country to keep inventory stable and allow for routine hospital operations in addition to beginning prospective triaging of blood orders to monitor and prioritize blood utilization [44].

In addition to applying the above-mentioned measures before and during blood donation, active post-donation information gathering, product tracing, and recall are suggested [45]. Registration of donor information and following up until the expiry date of the blood products has facilitated the isolation or urgent recall of blood products donated by individuals with suspected COVID-19 infection and the implementation of proactive measures such as the temporary isolation of blood for 14 days after collection and delaying its release for clinical use [19]. Donors are requested to report if they have any illness or close contact with a confirmed case prior to donation or if they were to be classified as a suspect case or diagnosed as COVID-19 after donation [46]. It is worth noting that it is optimal for blood services to receive the details of all confirmed COVID-19 cases from their health authorities and not solely rely on post-donation information provided by blood donors in order to be able to trace donors, recall any blood products not transfused, or apply a 3-month deferral for future donations for confirmed COVID-19 cases even though transfusion transmission of COVID-19 to recipients has not occurred so far [46].

\section{Plasma therapy}

\section{Treatment options in COVID-19}

Currently, there are no approved therapies for either the treatment or the prevention of COVID-19 [33]. Several treatment options are being explored and include vaccine development, convalescent plasma, interferon-based therapies, smallmolecule drugs, cell-based therapies, and monoclonal antibodies (mAbs) [2]. Lythgoe et al. have identified 344 interventional studies worldwide focusing on both preventative strategies and the treatment of patients with COVID-19 as of March 20, 2020 [33]. Treatment strategies under investigation can be divided into six main categories [33]:

1. Antiviral treatments including protease inhibitors (lopinavir/ ritonavir) and nucleotide analogues (remdesivir) in addition to several other antivirals used in the treatment of viral illnesses such as viral hepatitis C, influenza, Ebola, and HIV viruses

2. Antimalarial treatments including chloroquine and hydroxychloroquine

3. Immunomodulators including immunosuppressants such as corticosteroids, adalimumab (antiTNF), eculizumab (anti-C5), sarilumab (anti-IL-6), ixekizumab (anti-17A), and fingolimod (sphingosine-1-phosphate receptor modulator) or immunostimulants such as anti-PD-1 antibody camrelizumab and recombinant IL-2

4. Cell-based therapy such as mesenchymal stem cells (MSCs) which have immunomodulatory and tissue repair effects through the secretion of cytokines and growth factors

5. Plasma-based therapy

6. Other therapies: the anti-fibrotic/inflammatory agent pirfenidone and anti-angiogenic agents (bevacizumab and thalidomide)

Current efforts are also directed towards the rapid development of vaccines with the majority targeting the spike glycoprotein or S protein of coronaviruses [47]. Extensive vaccination is the only promising strategy to prevent the spread of COVID-19 pandemic; however, having a vaccine available to the general population might take some time [19]. To date, there are 18 biotechnology companies and universities in China working on COVID-19 vaccines among many others globally [23].

\section{Plasma therapy as a treatment option}

Convalescent (i.e., "immune") plasma refers to collected plasma following resolution of infection and development of antibodies, and it can be transfused as only a short-term treatment strategy to confer immediate immunity to susceptible individuals in the setting of post-exposure prophylaxis and/ or treatment of infectious diseases, including outbreaks of coronaviruses such as SARS-1 and Middle East Respiratory Syndrome (MERS) [48]. The use of convalescent plasma was first studied with SARS and Ebola in years 2003 and 2012, respectively, when plasma administered early after symptom 
onset in the treatment of SARS patients was shown to improve outcomes (OR 0.25) [49] unlike in Ebola virus disease where there was no significant improvement in survival [50]. Singlecenter studies evaluating the role of convalescent plasma in COVID-19 have shown that convalescent plasma therapy is well tolerated and could potentially improve the clinical outcomes through neutralizing viremia in severe COVID-19 cases and helping in achieving radiological resolution and improving survival; however, the optimal dose and clinical benefit need further investigation in larger well-controlled trials [48, 51]. On March 26, 2020, the US Food and Drug Administration (FDA) approved the use of convalescent plasma from patients who have recovered from COVID-19 to treat people who are critically ill after securing approval over the telephone [52]. Currently, the National Health Commission of China has asked patients to donate blood for the treatment of COVID19 infection in order to collect convalescent plasma within 2 weeks after recovery to ensure a high neutralization antibody titer [23], as studies have found that the titers of neutralizing antibodies reach their peak at 10 to 15 days after disease onset and remain stable thereafter in patients [53]. A meta-analysis published in May 2020 by Rajendran et al. has concluded that CPT therapy in COVID-19 patients appears safe, clinically effective, and reduces mortality [54]. On the other hand, a phase 3 clinical trial by Li et al. has shown that the use of convalescent plasma therapy added to standard treatment, compared with standard treatment alone, did not result in a statistically significant improvement in time to clinical improvement within 28 days among patients with severe or life-threatening COVID-19. More well-designed large multicenter clinical trials shall be conducted to better assess the role of convalescent plasma in COVID-19 patients [55].

Despite the promising results and the robust infrastructure blood banks globally have, regulatory and logistical challenges are inevitable and are related to the following: (1) donor eligibility: selected donors need to be virus-free at the time of blood collection given the potential risk to blood collections staff and other donors; (2) donor recruitment: patients who have recovered from COVID-19 can be recruited to serve as potential blood donors and outreaching potential donors is not considered a major problem; (3) testing: antibody testing is available through qualitative and quantitative assays, but the relationship between total COVID-19 antibodies and neutralizing anti-COVID-19 antibodies remains unclear although it is recommended to determine the titer of anti-COVID-19 IgG and to apply virus inactivation procedures strictly before the use of plasma; (4) collection: apheresis rather than whole blood donation is recommended to optimize the yield of convalescent plasma and is highly efficient where around 400$800 \mathrm{~mL}$ of plasma from a single apheresis donation can provide 2-4 units of convalescent plasma which can be either transfused or frozen within $24 \mathrm{~h}$ of collection; and finally (4) transfusion: the dosing of convalescent plasma is highly variable and it is suggested to transfuse one unit for postexposure prophylaxis and one to two units for treatment of severe infections $[19,48,56]$.

As for convalescent plasma transfusion safety, the risks to transfusion r4ecipients are likely to be no different from those of standard plasma [64]. Regarding non-infectious hazards of transfusion such as allergic transfusion reactions, transfusionassociated circulatory overload (TACO), and transfusionrelated acute injury (TRALI), the risk is considered low, but TRALI is the one to be of particular concern in severe COVID-19 patients given potential priming of the pulmonary endothelium [48]. Furthermore, treatment with human immunoglobulin or plasma has been associated with significantly increased same-day thrombotic event risk (0.04 to $14.9 \%)$ as shown in previous studies [57], indicating the potential value of evaluating the effectiveness of early intervention therapy with convalescent plasma in patients with acute respiratory distress as an effective therapy [58]. Since well-designed clinical trials to further evaluate the efficacy and safety of convalescent plasma therapy in patients with COVID-19 infection are ongoing, current recommendations insist that the convalescent plasma should be given only to patients with advanced disease and severe and critical cases of COVID-19 until more solid data on its effective and safety become available [19].

\section{Conclusion}

With the recent and rapid evolution of COVID-19 pandemic worldwide, various challenges have emerged. Transfusion medicine is one of the health fields highly affected where blood safety and blood product availability are real global challenges. COVID-19 pandemic has led to the development of new strategies at blood banks and hospitals in order to overcome the shortage in blood products. Hospitals are encouraged to have in place an emergency blood management plan in preparedness planning for sustainability and safety of blood supply [30]. As for the treatment of COVID-19, there are no therapeutic options proven to be effective, but multiple options are currently being studied, one of which is the use of convalescent plasma which can be considered a promising rescue option especially when dealing with severe COVID19 patients, keeping in mind the urgent need for randomized clinical trials.

Author Contribution Layal Al Mahmasanil and Mohammad Hassan Hodroj1 contributed equally to this work.

\section{Declarations}

Ethics Approval and Consent to Participate This article does not contain any studies with human participants performed by any of the authors. 
Conflict of Interest The authors declare that they have no conflict of interest.

\section{References}

1. de Wilde AH et al (2018) Host factors in coronavirus replication. Curr Top Microbiol Immunol 419:1-42

2. Vijaykrishna D, Smith GJ, Zhang JX, Peiris JS, Chen H, Guan Y (2007) Evolutionary insights into the ecology of coronaviruses. J Virol 81(8):4012-4020.https://doi.org/10.1128/JVI.02605-06. Erratum in: J Virol. 2007 Aug;81(15):8371

3. Weston S, Frieman MB (2020) COVID-19: Knowns, unknowns, and questions. mSphere 5(2)

4. de Wit E et al (2016) SARS and MERS: recent insights into emerging coronaviruses. Nat Rev Microbiol 14(8):523-534

5. Paules CI, Marston HD, Fauci AS (2020) Coronavirus infectionsmore than just the common cold. JAMA 323(8):707-708

6. Li Q et al (2020) Early transmission dynamics in Wuhan, China, of novel coronavirus-infected pneumonia. N Engl J Med 382(13): 1199-1207

7. Zhu N et al (2020) A novel coronavirus from patients with pneumonia in China, 2019. N Engl J Med 382(8):727-733

8. $\mathrm{Xu} \mathrm{X}$ et al (2020) Evolution of the novel coronavirus from the ongoing Wuhan outbreak and modeling of its spike protein for risk of human transmission. Sci China Life Sci 63(3):457-460

9. Jin $Y$ et al (2020) Virology, epidemiology, pathogenesis, and control of COVID-19. Viruses 12(4):372

10. Epidemiology Working Group for NCIP Epidemic Response, Chinese Center for Disease Control and Prevention (2020) The epidemiological characteristics of an outbreak of 2019 novel coronavirus diseases (COVID-19) in China. Zhonghua Liu Xing Bing Xue Za Zhi 41(2):145-151

11. Chen $\mathrm{N}$ et al (2020) Epidemiological and clinical characteristics of 99 cases of 2019 novel coronavirus pneumonia in Wuhan, China: a descriptive study. Lancet 395(10223):507-513

12. Guan WJ et al (2020) Clinical characteristics of coronavirus disease 2019 in China.4. N Engl J Med 382(18):1708-1720

13. Hoehl S et al (2020) Evidence of SARS-CoV-2 infection in returning travelers from Wuhan, China. N Engl J Med 382(13): $1278-1280$

14. Connell NT (2016) Transfusion medicine. Prim Care 43(4):651659

15. Franchini $\mathrm{M}$ et al (2019) Patient blood management: a revolutionary approach to transfusion medicine. Blood Transfus 17(3):191195

16. Mascaretti L, De Angelis V, Berti P (2020) The severe acute respiratory syndrome coronavirus 2 (SARS-CoV-2) pandemic and transfusion medicine: reflections from Italy. Blood Transfus 18(2):7778

17. Chang L, Yan Y, Wang L (2020) Coronavirus disease 2019: coronaviruses and blood safety. Transfus Med Rev 34(2):75-80

18. Lan L et al (2020) Positive RT-PCR test results in patients recovered from COVID-19. JAMA 323(15):1502-1503

19. Cai $\mathrm{X}$ et al (2020) Blood transfusion during the COVID-19 outbreak. Blood Transfus 18(2):79-82

20. Rabenau HF et al (2005) Stability and inactivation of SARS coronavirus. Med Microbiol Immunol 194(1-2):1-6

21. Darnell ME, Taylor DR (2006) Evaluation of inactivation methods for severe acute respiratory syndrome coronavirus in noncellular blood products. Transfusion 46(10):1770-1777

22. Rebulla P (2019) The long and winding road to pathogen reduction of platelets, red blood cells and whole blood. Br J Haematol 186(5): 655-667
23. Zhai $\mathrm{P}$ et al (2020) The epidemiology, diagnosis and treatment of COVID-19. Int J Antimicrob Agents 55(5):105955

24. Waheed U et al (2020) Detection of severe acute respiratory syndrome coronavirus 2 RNA in blood donations. J Lab Physicians 12(2):163-164

25. Stanworth SJ et al (2020) Effects of the COVID-19 pandemic on supply and use of blood for transfusion. Lancet Haematol 7(10): e756-e764

26. Chang L et al (2020) Severe acute respiratory syndrome coronavirus 2 RNA detected in blood donations. Emerg Infect Dis 26(7): $1631-1633$

27. WHO [Internet] Laboratory biosafety guidance related to the novel coronavirus (2019-nCoV). Available at: https://www.who.int/docs/ default-source/coronaviruse/laboratory-biosafety-novelcoronavirus-version-1-1.pdf. Accessed on 09/05/2020

28. CDC [Internet] Interim laboratory biosafety. guidelines for handling and processing specimens associated with coronavirus disease 2019 (COVID-19). Available at: https://www.cdc.gov/coronavirus/ 2019-nCoV/lab/lab-biosafety-guidelines.html. Accessed on: 09/05/ 2020

29. Gschwender AN, Gillard L (2017) Disaster preparedness in the blood bank. Am Soc Clin Lab Sci 30(4):250-257

30. Fan BE et al (2020) Blood and blood product use during COVID19 infection. Am J Hematol 95(7):E158-E160

31. Mohammadi S et al (2020) Coronavirus disease 2019 (COVID-19) and decrease in blood donation: experience of Iranian Blood Transfusion Organization (IBTO). Vox Sang 115(7):595-596

32. Shander A et al (2020) The essential role of patient blood management in a pandemic: a call for action. Anesth Analg 131(1):74-85

33. Lythgoe MP, Middleton P (2020) Ongoing clinical trials for the management of the COVID-19 pandemic. Trends Pharmacol Sci 41(6):363-382

34. Ramanathan K et al (2020) Blood transfusion strategies and ECMO during the COVID-19 pandemic \&\#x2013; Authors' reply. Lancet Respir Med 8(5):e41

35. Mueller MM et al (2019) Patient blood management: recommendations from the 2018 Frankfurt Consensus Conference. JAMA 321(10):983-997

36. Mo A et al (2017) Red cell transfusion thresholds in myelodysplastic syndromes: a clinician survey to inform future clinical trials. Intern Med J 47(6):695-698

37. Spahn DR et al (2019) Effect of ultra-short-term treatment of patients with iron deficiency or anaemia undergoing cardiac surgery: a prospective randomised trial. Lancet 393(10187):2201-2212

38. Weinkove R et al (2020) Managing haematology and oncology patients during the COVID-19 pandemic: interim consensus guidance. Med J Aust 212(10):481-489

39. Estcourt LJ et al (2017) Guidelines for the use of platelet transfusions. Br J Haematol 176(3):365-394

40. DeBaun MR (2020) Initiating adjunct low dose-hydroxyurea therapy for stroke prevention in children with SCA during the COVID19 pandemic. Blood 135(22):1997-1999

41. Spahn DR et al (2020) Patient blood management: effectiveness and future potential. Anesthesiology 133(1):212-222

42. Sadana D et al (2018) Promoting high-value practice by reducing unnecessary transfusions with a patient blood management program. JAMA Intern Med 178(1):116-122

43. Combes A et al (2018) Extracorporeal membrane oxygenation for severe acute respiratory distress syndrome. N Engl J Med 378(21): 1965-1975

44. Pagano MB et al (2020) Prepare to adapt: blood supply and transfusion support during the first 2 weeks of the 2019 novel coronavirus (COVID-19) pandemic affecting Washington State. Transfusion 60(5):908-911

45. Dodd RY, Stramer SL (2020) COVID-19 and blood safety: help with a dilemma. Transfus Med Rev 34(2):73-74 
46. Kwon SY et al (2020) Post-donation COVID-19 identification in blood donors. Vox Sang 115(8):601-602

47. Du L et al (2009) The spike protein of SARS-CoV-a target for vaccine and therapeutic development. Nat Rev Microbiol 7(3): 226-236

48. Bloch EM et al (2020) Deployment of convalescent plasma for the prevention and treatment of COVID-19. J Clin Invest 130(6):27572765

49. Mair-Jenkins J et al (2015) The effectiveness of convalescent plasma and hyperimmune immunoglobulin for the treatment of severe acute respiratory infections of viral etiology: a systematic review and exploratory meta-analysis. J Infect Dis 211(1):80-90

50. van Griensven J et al (2016) Evaluation of convalescent plasma for Ebola virus disease in Guinea. N Engl J Med 374(1):33-42

51. Kai Duan, Bende Liu, Cesheng Li et al (2020) The feasibility of convalescent plasma therapy in severe COVID- 19 patients: a pilot study. MedRxiv. The preprint server for health sciences. https://doi. org/10.1101/2020.03.16.20036145

52. Tanne JH (2020) Covid-19: FDA approves use of convalescent plasma to treat critically ill patients. BMJ 368:m1256

53. Wu F et al (2020) Neutralizing antibody responses to SARS-CoV-2 in a COVID-19 recovered patient cohort and their implications.
MedRxiv. The preprint server for health sciences. https://doi.org/ $10.1101 / 2020.03 .30 .20047365$

54. Rajendran $\mathrm{K}$ et al (2020) Convalescent plasma transfusion for the treatment of COVID-19: systematic review. J Med Virol 92(9): $1475-1483$

55. Li L et al (2020) Effect of convalescent plasma therapy on time to clinical improvement in patients with severe and life-threatening COVID-19: a randomized clinical trial. JAMA 324(5):460-470

56. Epstein J, Burnouf T (2020) Points to consider in the preparation and transfusion of COVID-19 convalescent plasma. Vox Sang 115(6):485-487

57. Menis M et al (2013) Hyperimmune globulins and same-day thrombotic adverse events as recorded in a large healthcare database during 2008-2011. Am J Hematol 88(12):1035-1040

58. Cunningham AC, Goh HP, Koh D (2020) Treatment of COVID19: old tricks for new challenges. Crit Care 24(1):91

Publisher's note Springer Nature remains neutral with regard to jurisdictional claims in published maps and institutional affiliations. 